\title{
¿Segregación Escolar por Nivel Socioeconómico o por Nivel de Estudios de los Padres?
}

\author{
School Segregation by Socioeconomic Status or by Parental \\ Education?
}

\author{
F. Javier Murillo * \\ Raquel Graña \\ Universidad Autónoma de Madrid, España
}

\begin{abstract}
Con esta investigación se pretende determinar si es más adecuado considerar la segregación escolar por nivel socioeconómico de las familias o por nivel de estudios de los padres, en términos de cuál alcanza valores más altos y cuál tiene una mayor relación con el rendimiento estudiantil. Para ello, se realiza una explotación especial de la base de datos de la prueba de evaluación colombiana SABER 2016, de carácter censal. Se analizan 646.413 estudiantes de $5^{\circ}$ grado escolarizados en 14.630 escuelas de Colombia. En primer lugar, se estima el Îndice de Segregación de Gorard y el Índice de Raíz Cuadrada de Hutchens para nueve grupos según el nivel de estudios de los padres y otros, con tamaño equivalente, según la segregación por nivel socioeconómico. En segundo término, se estiman modelos multinivel de dos niveles (escuela y estudiantes). Los resultados muestran, por un lado, que para todos los grupos minoritarios y en ambos índices, la segregación escolar por nivel socioeconómico es mayor que segregación por estudios de los padres. Y, por otro, que, aunque ambas consideraciones de segregación afectan al rendimiento, la segregación por nivel socioeconómico muestra una relación mayor. De esta forma se concluye que, tal y como se venía haciendo, es correcto estimar la segregación por nivel socioeconómico, más que por nivel de estudios de los padres.
\end{abstract}

Descriptores: Segregación escolar; Nivel socioeconómico; Nivel de estudios de los padres; Rendimiento académico; Educación primaria.

This research aims to determine whether it is more appropriate to consider school segregation by socioeconomic status of families or by parents' educational level, both in terms of which is higher, and which has a greater relationship with student performance. We did a special exploitation of the census nature database of the Colombian evaluation test SABER 2016. A total of 646,413 5th grade students enrolled in 14,630 schools are analyzed. First, the Gorard Segregation Index and the Hutchens Square Root Index are estimated for nine groups according to the level of education of the parents and others, with equivalent size, according to segregation by socioeconomic level. Second, multilevel models of two levels (school and students) are estimated. On the one hand, the results show that for all minority groups and in both indices, school segregation by socioeconomic level is greater than segregation by parent's educational level. And, on the other hand, that, although both segregation considerations affect student performance, segregation by socioeconomic level shows a greater relationship. In this way, it is concluded that it is correct to estimate segregation by socioeconomic level at is has been done, rather than by parents' educational level.

Keywords: School segregation; Socioeconomic status; Parental educational level; Educational achievement; Primary education.

*Contacto: javier.murillo@uam.es

ISSN: $1696-4713$

www.rinace.net/reice/

revistas.uam.es/reice
Recibido: $\quad 3$ de marzo 2020

$1^{\text {a }}$ Evaluación: 27 de junio 2020

$2^{\text {a }}$ Evaluación: 15 de agosto 2020

Aceptado: 29 de agosto 2020 


\section{Introducción}

Hace casi dos décadas, Gorard y Taylor (2002) afirmaron que existe en la literatura especializada una multiplicidad de interpretaciones sobre el término "segregación". Pasado ese tiempo, la situación no ha cambiado en lo esencial: no se cuenta con una definición clara, operativa y consensuada sobre que es segregación ni, por supuesto, cómo estimar su magnitud. Las consecuencias de este hecho son mucho más devastadoras de lo que puede derivarse de un simple debate académico; implican la dificultad de poder denunciar con rotundidad un hecho que trae implicaciones directas en la equidad de la educación y en la construcción de una sociedad más justa y sin exclusiones.

Efectivamente, a la falta de una definición consensuada del término "segregación escolar" se suma la multiplicidad de índices para medirla, las diferentes variables criterios que se usan para los distintos tipos de segregación o la conformación de los grupos minoritarios. Este hecho hace que sea muy complicado comparar las diferentes investigaciones para estudiar los avances en la segregación, pero sobre todo complejiza enormemente la comunicación a la sociedad y a las personas que asumen responsabilidades políticas en educación. De esta forma, desde la investigación no se facilita en la manera que sería deseable el diseño de políticas públicas para luchar contra esta lacra social y educativa.

En una pasada investigación, buscando mejorar los aspectos metodológicos de la investigación sobre segregación escolar, se abordó el tema de la elección del grupo minoritario para los casos en los que la variable criterios fuera dependiente y hubiera que dicotomizarla (Murillo y Martinez-Garrido, 2019). Entonces se propuso la estimación de un "perfil de segregación escolar" como una forma de comprender mejor la segregación y ayudar a la toma de decisiones política.

Este trabajo aborda otro elemento metodológico y se centra en contestar de la siguiente pregunta de investigación: ¿es más adecuado estimar la segregación escolar por nivel socioeconómico de la familia o por nivel de estudios de los padres? Su justificación recae en que existe poca investigación sobre segregación escolar con el nivel de estudios del padre y de la madre como variable criterio, pero, como luego se verá, esta variable tiene una fuerte influencia sobre el aprendizaje de los estudiantes, más que el nivel de ingresos de los padres.

\section{Revisión de la literatura}

Efectivamente, la estimación de la magnitud de la segregación escolar conlleva tener que tomar una serie de decisiones de carácter técnico y metodológico que puede hacer que los resultados sean completamente diferentes por un camino u otro. Así, hay que plantearse, al menos, cinco decisiones: 1) qué definición de segregación se opta, 2) qué tipo de segregación, 3) con qué variable criterio, 4) qué índice se usa, y 5) qué grupo minoritario se elige para su estimación. En esta investigación se aborda directamente la segunda decisión y, con ello, la tercera: qué tipo de segregación y qué variable criterio.

Sin entrar en detalles, es posible hablar de que hay tres grandes concepciones de segregación escolar. En primer lugar, estaría la segregación escolar como la distribución desigual de estudiantes en las escuelas en función de sus características personales o sociales o de sus condiciones. Esta definición se corresponde a la dimensión de uniformidad de Massey y Denton (1988). A pesar de su aparente sencillez, puede ser operativizada de 
distintas maneras. Así, por ejemplo, se entiende como la proporción de estudiantes que tendrían que cambiar de escuela para que existiera una distribución uniforme de desventaja entre las escuelas dentro del área de análisis; que sería lo que mide el Índice de Didimilitud (ID). Pero también: la diferencia entre la proporción de un grupo minoritario en una institución y la proporción de todos los miembros del grupo en la misma institución, que se correspondería al Índice de Segregación de Gorard (ISG). O también la segregación escolar entendida como la distancia entre la media geométrica de las participaciones de estudiantes de distinto grupo minoritario en ausencia de la segregación y de la media geométrica de las participaciones reales (el alejamiento de cada escuela de la igualdad distributiva), que es lo que identifica el Índice de Raíz Cuadrada de Hutchens (IH).

Otra concepción diferente de segregación es la que hace referencia al grado de aislamiento/exposición de un sujeto respecto a su grupo minoritario. $\mathrm{O}$, en palabras de Croxford y Raffe (2013, p. 178), "el grado en el que los miembros de un grupo minoritario están expuestos sólo entre sí y, por lo tanto, aislados de los miembros de su grupo complementario". Es lo que mide el Índice de Aislamiento (IA) y se corresponde con la dimensión de exposición de Massey y Denton (1988).

Por último, sería posible considerar la segregación como "la situación de homogeneidad social intra-escolar y de heterogeneidad social inter-escolar" (Benito y González-Baetbó, 2007, p. 41). Es decir, el porcentaje de varianza de desigualdad inter escolar respecto al total, que es lo que estima el Índice de Inclusión Social (ISS) (Murillo, 2016; OECD, 2010).

Ante tanta variedad, es muy habitual que los diferentes trabajos, más que optar por una definición y un índice, ofrezcan diferentes estimaciones. Ello le dota a los trabajos de mayor coherencia interna, pero no contribuye a cerrar el debate y decantarse por una alternativa.

Sea como fuere, en todas estas definiciones se habla de "grupo minoritario" conformado por sus "características sociales o personales". Según cual sean esas características, se estará hablando de un tipo u otro de segregación escolar. De esta forma es posible distinguir, en primer lugar, la segregación escolar por origen étnico. Como es conocido, las primeras investigaciones realizadas sobre segregación escolar surgen en Estados Unidos, tras el fallo que la Corte Suprema que declara inconstitucionales las leyes estatales que establecían escuelas públicas separadas para estudiantes "negros" y "blancos" en el llamado caso Brown contra el Consejo de Educación de Topeka (Coleman, 1975). De esta forma, los primeros pasos en la investigación sobre segregación escolar giraron en torno a la segregación racial, antes centrada en la segregación de afrodescendientes y, actualmente, en la segregación de otras minorías, como las personas procedentes de Latinoamérica (p. ej. Fiel y Zhang, 2018; Fuller et al., 2019; Santiago, 2019).

En Europa la preocupación original procedió de la concentración de estudiantes de origen extranjero en determinados centros educativos, es la llamada segregación escolar por origen nacional (Bonal, Zancajo y Scandurra, 2019; Karsten, 2010; Murillo, MartínezGarrido y Belavi, 2017; Nordin, 2013). Aunque con un desarrollo más tardío, existe una especial preocupación por la segregación escolar en función del nivel socioeconómico de las familias. Uno de los trabajos pioneros sobre esta temática es The Truly Disadvantaged de William J. Wilson (1987) que estudia las desventajas de la concentración en escuelas de las familias de clases sociales más desfavorecidas. Desde entonces se han desarrollado en todo el mundo numerosos trabajos que abordan este tipo de segregación (p. ej., Gorard 
y Siddiqui, 2018; Janmaat, 2020; Krüger, 2019; Murillo y Martínez-Garrido, 2018; Murillo, Duk y Martínez-Garrido, 2018; OECD, 2019; Zhou, Cai y Wang, 2016).

Menos son las investigaciones que han considerado el nivel de estudios del padre y de la madre para estimar la segregación escolar. Entre ellos, destacan los trabajos sobre la segregación escolar en Suecia, un país con una fuerte preocupación por la equidad de sus escuelas. Así es posible encontrar los estudios de Böhlmark, Holmlund y Lindahl (2016), Yang Hansen y Gustafsson (2016a), de Kornhall y Bender (2019) o de Ortiz y Dehon (2008), por poner algunos ejemplos. Pero también han usado este enfoque estudios que analizan la segregación escolar en Países Bajos (Gramberg, 1998), en Grecia (Maloutas et al., 2013), en Brasil (Bartholo, 2013), en Rusia (Ivaniushina, Makles, Schneider y Alexandrov, 2019), en Paquistán (Siddiqui, 2017), o en España (Benito y González-Baetbó, 2007; Mancebón-Torrubia y Pérez-Ximénez, 2010), por poner algunos ejemplos.

La opción de usar el nivel de estudio de los progenitores como variable criterio para la consideración de segregación escolar está más que justificada. El nivel de estudios del padre y de la madre forma parte de lo que Bourdieu (1973) denominó "capital cultural" con una clara incidencia en la reproducción social (Davies y Rizk, 2018). Así, está largamente demostrada su fuerte incidencia en el rendimiento académico de los estudiantes (p. ej., Bodovski, 2010; Brese y Mirazchiyski, 2013; Davis-Kean, 2005; Desforges y Abouchaar, 2003; Erola, Jalonen y Lehti, 2016; Gorard y See, 2013; Hansen y Gustafsson, 2016; Fajardo Bullón et al., 2017; Murillo y Román, 2011, Mullis y Jenkins, 1990; Silles, 2011; Sirin, 2005; White, 1982). Esta incidencia del nivel de estudios de los padres sobre el rendimiento se explica por sus creencias, valores, expectativas, actitudes y comportamientos (Gustafsson, Yang Hansen y Rosén, 2013). Así los padres con mayor formación tendrían expectativas más altas, a la vez que adaptarían sus expectativas al rendimiento de sus hijos; también en estos hogares el clima en el hogar sería más positivo y dirigido a lo académico. Los padres con menores estudios, por su parte, tendrían unas expectativas más bajas o poco realistas.

Pocos trabajos han estimado de forma simultánea la segregación escolar por nivel socioeconómico y por nivel de estudios que permita comparar ambos resultados. Mancebón-Torrubia y Pérez-Ximénez (2010), para comenzar, realizan un estudio para medir la segregación escolar en España y sus Comunidades Autónomas en función de la titularidad de centro y cómo la segregación se relaciona con el rendimiento de los y las estudiantes. Los autores, usando los datos de PISA-2006, estiman la magnitud de la segregación de escolar en España mediante el Índice de Disimilitud para cinco variables criterio: nivel de estudios de los padres, tipo de trabajo de los padres, status de inmigración, ocupación de los padres y nivel socioeconómico y cultural. Los resultados muestran que se da una mayor segregación por origen nacional, a continuación, se encontraría la segregación por tipo de trabajo de los padres, después con nivel socioeconómico y cultural, seguido de estudios de los padres y, el menor, por su ocupación profesional. Aunque con diferencias según si el centro es público o privado. Estos resultados son análogos a los hallados por Benito y González-Baetbó (2007) que, analizando diversos municipios de Cataluña, encontraron que la segregación escolar por origen nacional era superior a la segregación escolar por estudios de los padres, aunque con diferencias entre municipios.

Resultados similares fueron hallados por Yang Hansen y Gustafsson (2016a) en un estudio sobre la evolución en la segregación escolar y elección de centro en diferentes tipos de 
municipios, entre 1998 y 2011, en Suecia. Los autores exploran los cambios que se han producido entre las escuelas con respecto a la educación de los padres y las madres, los antecedentes de migración y el rendimiento en diferentes municipios a lo largo del tiempo. Encuentran, en primer lugar, que el aumento de la elección de las escuelas se asocia con una mayor segregación escolar con respecto al nivel de educación de los padres. Sin embargo, existe un mayor aumento de la segregación escolar por origen nacional. Este trabajo se complementa con el de Böhlmark, Holmlund y Lindahl (2016), también en Suecia. Sus hallazgos apuntan a que la mayor segregación escolar se da por origen nacional, seguida por la segregación escolar por nivel de estudios de los padres y, por último, la segregación por nivel de ingreso de los padres de los estudiantes.

Un último ejemplo lo aporta Bartholo (2014), quien analiza la movilidad de estudiantes dentro de la red de escuelas públicas de Río de Janeiro y su relación con la segregación escolar tanto por nivel socioeconómico, antecedentes étnicos como por la educación de madres y padres. Los resultados apuntan a que la segregación escolar por nivel de estudios de las familias, medido con el Índice de Segregación de Gorard, presenta valores más altos que la segregación por nivel socioeconómico. Así, para estudiantes de quinto curso del año 2009 estima que la segregación según el nivel de estudios de las familias es de un 0,24, mientras que índice de segregación escolar por nivel socioeconómico, en el mismo curso y año, es del 0,19.

En esta investigación se pretende determinar qué tipo de segregación escolar es más adecuada para conocer la situación de inequidad de los sistemas educativos, si por nivel socioeconómico de las familias o por nivel de estudios de los padres. Para ello se alcanzar dos objetivos: en primer lugar, determinar la magnitud de la segregación escolar por nivel educativo de las familias y por nivel socioeconómico de las mismas para conocer cuál alcanza valores más altos y, por otro lado, estimar cuál de los dos tipos de segregación escolar incide más en el rendimiento de los estudiantes.

\section{Método}

Para dar respuesta a los objetivos se ha desarrollado una explotación especial de la base de datos de SABER 2016, correspondiente a la evaluación de estudiantes colombianos de $5^{\text {a }}$ grado. Se optó por utilizar estos datos por varias razones: por ser datos censales, lo que evita sesgos generados por el uso de muestras, por aportar información diferenciada sobre el estatus socioeconómico (ESE) -a partir de las posesiones, los ingresos y las ocupaciones de los padres- y el nivel de estudios del padre y el de la madre, con lo que es posible hacer esa diferenciación, y, por último, por tener datos sobre el rendimiento de estudiantes, lo que permite alcanzar el segundo de los objetivos.

La prueba SABER, diseñada por el Instituto Colombiano para la Evaluación de la Educación, es una prueba para valorar el desarrollo de competencias básicas en los estudiantes de Educación Básica de $3^{\circ}, 5^{\circ}$ y $9^{\circ}$ curso, a través de cuatro pruebas -Lenguaje, Ciencias Sociales, Ciencias Naturales y Matemáticas- y un cuestionario de contexto que los estudiantes deben responder. La muestra de estudio la conforman 646.413 estudiantes de $5^{\circ}$ grado matriculados en 14.630 escuelas de los 32 departamentos en los que se organiza Colombia y el Distrito Capital de Bogotá.

Para alcanzar el primer objetivo, determinar la magnitud de la segregación escolar por nivel educativo de las familias y por nivel socioeconómico de las mismas, se estiman 
mediante el Índice de Segregación de Gorard (ISG) y el Índice de Raíz Cuadrada de Hutchens (IH). La razón de usar el primero es que frente a otros como el de Disimilitud, del que procede, es que no se ve afectado por el tamaño del grupo minoritario (Gorard y Taylor, 2002), además de haberse convertido en los últimos años en la opción más habitual por los investigadores. La razón de elegir el Índice de Raíz Cuadrada de Hutchens (Hutchens, 2001, 2004) recae en que, frente a otros índices como el de Disimilitud, de Aislamiento, de Brecha por Centiles o de Inclusión Socioeconómica (Murillo, 2016) -por citar los más populares-, es el único que tiene la propiedad de descomposición aditiva de sus elementos. De tal forma que es posible determinar la segregación de cada escuela individual, siendo el único que puede usarse, por tanto, para alcanzar el segundo objetivo.

Para tener una imagen lo más completa posible se ha optado por estimar la segregación con ambos índices para nueve grupos minoritarios: ni el padre ni la madre tienen estudios completos de primaria (e1), el padre no tiene estudios completos de primaria (e2), la madre no tiene estudios completos de primaria (e3), ambos progenitores tienen estudios de primaria o menos (e4), el padre tiene estudios de primaria o menos (e5) y la madre tiene estudios completos de primaria o menos (e6), la madre tiene estudios universitarios (e7), el padre tiene estudios universitarios (e8) y ambos progenitores los tienen (e9). Y, para contrastar con la magnitud de la segregación por nivel socioeconómico sin que influya el tamaño del grupo minoritario, se ha estimado con nueve grupos minoritarios del mismo tamaño que los antes mostrados. Así se ha calculado la segregación para el 10,64\% de los estudiantes de familias con menor nivel socioeconómico (c1, P10,64), el 16,35\% (c2, P 16,35), el 20,28\% (c3, P20,28), el 25,19\% (c4, P25,19), el 32,19\% (c5, P32,19) y el 37,18\% (c6, P37,18), y el 13,30\% de los estudiantes de familias con mayor nivel socioeconómico (c7, P86,7), el 12,10\% (c8, P87,93) y el 7,76\% (c9, P92,24).

Para responder al segundo objetivo, estimar cuál de los dos tipos de segregación incide más en el rendimiento de los y las estudiantes, se han calculado Modelos Multinivel de dos niveles (escuela y estudiante), con las siguientes variables:

- Variables dependientes: Rendimiento en Lengua y rendimiento en Matemáticas. Ambas fueron re-escaladas para esta investigación, de tal forma que la media es de 500 y la desviación típica de 100.

- Variable de ajuste: Nivel socioeconómico de la familia del estudiante. Variable estimada por el ICFES a partir de un conjunto de variables relativas a las posesiones familias, los ingresos y el trabajo del padre y la madre Variable tipificada en origen.

- Variables explicativas: Magnitud de la segregación de cada escuela por nivel de estudios de los padres y por nivel socioeconómico estimado a través del índice de Raíz Cuadrada de Hutchens, con los 18 grupos minoritarios antes descritos (nueve para la segregación por nivel educativo y otros tantos para la segregación por nivel socioeconómico).

Para estimar la relación entre rendimiento en cada una de las dos áreas (lengua y matemática) y las diferentes consideraciones de segregación, controlando las variables que pueden incidir en la relación se utilizaron Modelos Multinivel de dos niveles: escuela y estudiante. El proceso de modelado multinivel y para cada uno de los rendimientos, es el siguiente: 
Modelo 1, sólo con las variables de ajuste:

$$
\operatorname{Rend}_{i j}=\beta_{0 j}+\beta_{1 j} S E S_{i j}+\varepsilon_{i j}
$$

donde $\beta_{0 j}=\beta_{0}+\mu_{0 j} \quad \mu_{0 j k} \sim N\left(0, \sigma_{\mu 0}^{2}\right) \quad \varepsilon_{i j k} \sim N\left(0, \sigma_{\varepsilon}^{2}\right)$

Donde, para cada estudiante "i” y escuela “j”, $\beta_{0}$ representa la puntuación media del rendimiento en cada una de las dos áreas estudiadas, $\beta_{1 j}$ es la pendiente de la variable de control, y $\beta_{2 j}$ es la pendiente de las 18 variables de segregación. $\mu_{0 j}$ es el error asociado a cada escuela y $\varepsilon_{i j}$ es el error asociado cada estudiante.

Modelo 2, añadiendo individualmente cada una de las 18 variables explicativas $\left(S e g \_k\right)$ al modelo de ajuste (modelo 1):

$$
\operatorname{Rend}_{i j}=\beta_{0 j}+\beta_{1 j} S E S_{i j}+\beta_{2} \operatorname{Seg}_{-} k_{j}+\varepsilon_{i j}
$$

donde $\beta_{0 j}=\beta_{0}+\mu_{0 j} \quad \mu_{0 j k} \sim N\left(0, \sigma_{\mu 0}^{2}\right) \quad \varepsilon_{i j k} \sim N\left(0, \sigma_{\varepsilon}^{2}\right)$

Modelo 3, final, con todas las variables de forma simultánea.

$$
\operatorname{Rend}_{i j}=\beta_{0 j}+\beta_{1 j} S E S_{i j}+\beta_{2} \operatorname{Seg}_{-} 1_{j}+\beta_{3} \operatorname{Seg}_{-} 2_{j}+\cdots+\beta_{\mathrm{k}+1} \operatorname{Seg}_{-} k_{j}+\varepsilon_{i j}
$$

donde $\beta_{0 j}=\beta_{0}+\mu_{0 j} \quad \mu_{0 j k} \sim N\left(0, \sigma_{\mu 0}^{2}\right) \quad \varepsilon_{i j k} \sim N\left(0, \sigma_{\varepsilon}^{2}\right)$

Para determinar el peso de la influencia de la segregación escolar por nivel socioeconómico para cada uno de los grupos minoritarios y compararlo con el peso de la incidencia del ISE se estima el porcentaje de varianza del rendimiento en el nivel escuela explicada por cada una de las variables explicativas, y el total, y cada uno de los tres rendimientos analizados.

\section{Resultados}

De acuerdo con los objetivos planteados, se presentan los resultados en dos bloques, en primer lugar, se aborda la estimación de la magnitud por nivel socioeconómico y por nivel de estudios de los padres para conocer cuál de esos tipos genera una mayor segregación y, en segundo término, se analiza cuál de los dos tipos incide más en el rendimiento académico del estudiante.

\section{1. ¿Qué genera mayor segregación escolar?}

El primero de los objetivos es determinar qué tipo de segregación escolar, por nivel educativo de los padres o por su nivel socioeconómico, genera una mayor segregación. Para ello, se recuerda, se estima la segregación para nueve grupos minoritarios respecto al nivel educativo del padre y de la madre y se compara con otros tantos grupos del mismo tamaño, pero relativos al nivel socioeconómico de las familias. Se calcula tanto para el Índice de Segregación de Gorard, el más habitual, como para el Índice de Raíz Cuadrada de Hutchens.

Los resultados obtenidos de la estimación del Índice de Segregación de Gorard son bien nítidos (cuadro 1): la segregación escolar por nivel socioeconómico es más alta en todos y cada uno de los grupos minoritarios que la segregación escolar por nivel de estudios de los progenitores. Efectivamente, la segregación escolar considerando como grupo minoritario que ninguno de ambos progenitores tenga estudios completos de primaria es de 0,42. Dado que el 10,64\% de estudiantes están en esa situación se estima la segregación 
escolar por nivel socioeconómico para el grupo minoritario del 10,64\% de estudiantes de familias con menos estatus socioeconómico (ESE). En este caso, el resultado es de 0,55, considerablemente mayor respecto al nivel de estudios de los progenitores. Este mismo cálculo se repitió para cada uno de los 8 grupos minoritarios restantes y en todos los casos la segregación por nivel socioeconómico fue más alta. La distancia mayor es con el padre con estudios completos de primaria $(16,35 \%$ de los estudiantes) con 0,14 puntos de diferencia $(0,45$ y 0,31$)$, y la menor con ambos padres con la primaria completa o menos, con 0,07 puntos de diferencia $(0,41$ y 0,34$)$.

Cuadro 1. Magnitud de la segregación escolar por estudios de los padres y por nivel socioeconómico para seis grupos minoritarios equivalentes. Índice de Segregación de Gorard (ISG)

\begin{tabular}{lc|cc}
\hline \multicolumn{1}{c|}{ SEGREGACIÓN POR NIVEL DE ESTUDIOS } & ISG & $\begin{array}{c}\text { SEGREGACIÓN } \\
\text { SOCIOECONóMICA }\end{array}$ & ISG \\
\hline Padre y madre sin estudios completos de Primaria & 0,4189 & $\mathrm{P} 10,64$ & 0,5526 \\
Padre sin estudios completos de Primaria & 0,3135 & $\mathrm{P} 16,35$ & 0,4524 \\
Madre sin estudios completos de Primaria & 0,3242 & $\mathrm{P} 20,28$ & 0,4259 \\
\hline Padre y madre hasta Primaria o menos & 0,3429 & $\mathrm{P} 25,19$ & 0,4103 \\
Padre con Primaria o menos & 0,3247 & $\mathrm{P} 32,10$ & 0,4028 \\
Madre hasta Primaria o menos & 0,3551 & $\mathrm{P} 37,18$ & 0,4024 \\
\hline Madre estudios universitarios acabados & 0,4597 & $\mathrm{P} 86,70$ & 0,5539 \\
Padre estudios universitarios acabados & 0,4529 & $\mathrm{P} 87,93$ & 0,5709 \\
Madre y padre con estudios universitarios acabados & 0,5658 & $\mathrm{P} 92,25$ & 0,6417 \\
\hline
\end{tabular}

Fuente: Elaboración propia a partir de datos de SABER 2016.

Como parece razonable, los resultados son análogos si se estima la magnitud de la segregación escolar con el índice de Raíz Cuadrada: para todos los casos la segregación socioeconómica es mayor que la segregación por estudios de los progenitores (cuadro 2).

Cuadro 2. Magnitud de la segregación escolar por estudios de los padres y por nivel socioeconómico para seis grupos minoritarios equivalentes. Índice de Raíz Cuadrada de Hutchens (IH)

\begin{tabular}{lc|cc}
\hline \multicolumn{1}{c|}{ SEGREGACIÓN POR NIVEL DE ESTUDIOS } & IH & $\begin{array}{c}\text { SEGREGACIÓN } \\
\text { SOCIOECONóMICA }\end{array}$ & IH \\
\hline Padre y madre sin estudios completos de primaria & 0,2275 & $\mathrm{P} 10,64$ & 0,3587 \\
Padre sin estudios completos de primaria & 0,1850 & $\mathrm{P} 16,35$ & 0,3215 \\
Madre sin estudios completos de primaria & 0,1698 & $\mathrm{P} 20,28$ & 0,3070 \\
\hline Padre y Madre hasta Primaria o menos & 0,2012 & $\mathrm{P} 25,19$ & 0,2947 \\
Padre con Primaria o menos & 0,1790 & $\mathrm{P} 32,10$ & 0,2891 \\
Madre hasta Primaria o menos & 0,1700 & $\mathrm{P} 37,18$ & 0,2905 \\
\hline Madre estudios universitarios acabados & 0,2680 & $\mathrm{P} 86,70$ & 0,3892 \\
Padre estudios universitarios acabados & 0,2597 & $\mathrm{P} 87,93$ & 0,4012 \\
Madre y Padre con estudios universitarios acabados & 0,3653 & $\mathrm{P} 92,25$ & 0,4574 \\
\hline
\end{tabular}

Fuente: Elaboración propia a partir de datos de SABER 2016.

Efectivamente, comparando grupos equiparables en tamaño se encuentra que siempre la segregación por nivel socioeconómico es mayor, con diferencias que van desde los 0,1415 puntos menos en la situación en el que el padre tiene estudios universitarios, hasta los 0,0921 en lo que ambos progenitores tienen estudios universitarios completos. 
Aunque los resultados del uso de ambos índices son similares en lo referente al objetivo de esta investigación, un sencillo gráfico de dispersión muestra que su comportamiento es bastante diferente (gráfico 1). Así, la correlación entre la segregación por nivel de estudios y por nivel socioeconómico es de 0,667 con el Índice de Segregación de Gorard y de 0,833 para el Índice de Raíz Cuadrada de Hutchens (ambas correlaciones bivariadas estimadas con el Rho de Spearman).
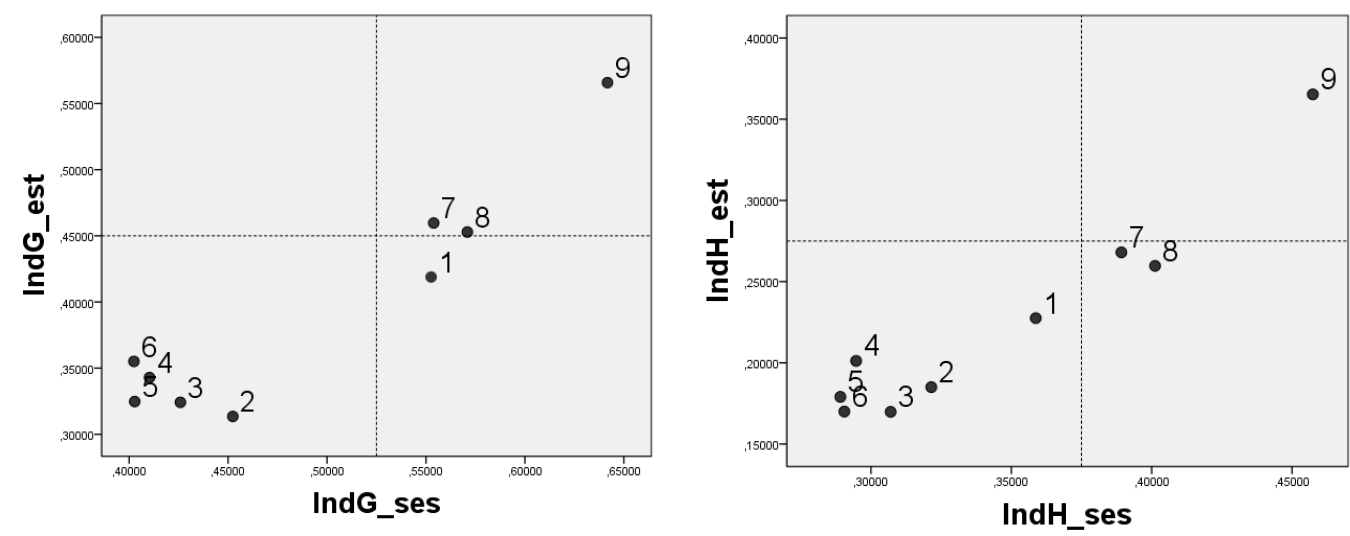

Gráfico 1. Relación entre la magnitud de la segregación escolar por nivel de estudios y nivel socioeconómico con el Índice de Gorard y con el Índice de Raíz Cuadrada Fuente: Elaboración propia a partir de datos de SABER 2016.

En todo caso, se puede concluir con toda claridad que hay una mayor segregación escolar por nivel socioeconómico que por nivel de estudios de los padres.

\section{2. ¿Qué segregación escolar incide más en el rendimiento?}

El segundo objetivo es determinar qué influye más en el rendimiento académico, si la segregación por nivel estudios de los progenitores o la segregación socioeconómica. Para ello se han estimado dos modelos multinivel, uno para cada área académica (rendimiento en Lengua y en Matemática), con las 18 estimaciones de segregación utilizando el Índice de Raíz Cuadrada de Hutchens (nueve para los diferentes grupos de nivel de estudios del padre y de la madre y otros tantos para sus análogos de segregación socioeconómica). A partir del porcentaje de varianza del rendimiento explicado por cada tipo de segregación se tendrá una estimación de qué tipo de segregación está más relacionada con el rendimiento y, con ello, es más adecuada.

En los cuadros 3 y 4 se presenta el resumen de los procesos de modelización donde, por razones de espacio y claridad, solo se ha incluido el porcentaje de la varianza de la escuela de cada rendimiento explicada por cada variable independiente respecto al modelo ajustado. Con esta información es suficiente para el objetivo de esta investigación, aunque se puede encontrar el proceso detallado de modelado multinivel en el anexo.

Las 18 variables independientes (explicativas), cada una con una forma diferente de medir la segregación escolar, hace una aportación estadísticamente significativa al modelo ajustado con el rendimiento en Lenguaje como variable independiente. Es decir, tanto la segregación escolar por nivel socioeconómico como por estudios de los padres incide en el rendimiento en Lenguaje. De ellas, las que tienen como grupo minoritario por debajo de la media (e1 a e6 y c1 a c6) su aportación es negativa, y los que están por encima de la 
media, la aportación es positiva. Es decir, los niños y niñas en escuelas con alta concentración de estudiantes de menor nivel socioeconómico y menos nivel de estudios de los padres ven perjudicado su rendimiento frente a los que no están en esas escuelas. Si, por el contrario, estudian en una escuela donde la segregación se produce por tener mayor presencia de alumnos con familias de mayor nivel socioeconómico o de estudios, entonces su rendimiento es mayor. Todo ello habiendo controlado el nivel socioeconómico del estudiante.

Cuadro 3. Resumen de resultados de los procesos de Modelado multinivel sobre la incidencia de la segregación escolar por nivel educativos del padre y de la madre del estudiante y de la segregación por nivel socioeconómico en el rendimiento en Lengua

\begin{tabular}{lc|lc}
\hline \multicolumn{1}{c|}{ SEGREGACIÓN POR NIVEL DE ESTUDIOS } & $\begin{array}{c}\text { \% VAR. } \\
\text { EXPLICADA }\end{array}$ & $\begin{array}{c}\text { SEGREGACIÓN } \\
\text { SOCIOECONÓMICA }\end{array}$ & $\begin{array}{c}\text { \% VAR. } \\
\text { EXPLICADA }\end{array}$ \\
\hline Madre y padre sin est. completos de Primaria & 1,87 & P10,64 & 3,36 \\
Padre sin estudios completos de Primaria & 2,78 & P16,35 & 4,22 \\
Madre sin estudios completos de Primaria & 2,35 & P20,28 & 4,86 \\
\hline Madre y padre hasta Primaria o menos & 2,12 & $\mathrm{P} 25,19$ & 5,72 \\
Padre con Primaria o menos & 3,81 & $\mathrm{P} 32,10$ & 7,17 \\
Madre hasta Primaria o menos & 3,25 & $\mathrm{P} 37,18$ & 8,29 \\
\hline Madre estudios universitarios acabados & 12,14 & $\mathrm{P} 86,70$ & 12,74 \\
Padre estudios universitarios acabados & 12,66 & $\mathrm{P} 87,93$ & 12,33 \\
Madre y padre con est. universitarios acabados & 10,17 & $\mathrm{P} 92,25$ & 10,35 \\
\hline
\end{tabular}

Fuente: Elaboración propia a partir de datos de SABER 2016.

Si se compara el peso, estimado a través del porcentaje de varianza explicada, que tiene cada una de las consideraciones de segregación se observa (cuadro 3) que la segregación por nivel socioeconómico es siempre superior (o casi siempre) que la segregación por nivel de estudios, confrontando grupos análogos. Sin embargo, es muy interesante verificar que la diferencia varía en función del nivel de estudios de los padres considerado. Efectivamente, encontramos que:

- Para la segregación de estudiantes donde al menos uno de los progenitores no tiene estudios completados de primaria, que se corresponde con los colectivos de muy bajo nivel socioeconómico, el porcentaje explicado por las distintas medidas de segregación por nivel de estudios es, de promedio, 1,8 puntos porcentuales más bajo que sus correspondientes medidas de segregación por nivel socioeconómico.

- Para la segregación escolar con los grupos minoritarios de estudiantes con padres y madres donde uno de los dos tenga estudios completos de primaria o menos, la diferencia con sus correspondientes grupos minoritarios en nivel socioeconómico es del 4,0\%.

- Por último, para los grupos donde al menos uno de los progenitores tiene estudios universitarios la diferencia es de apenas $0,15 \%$. Incluso con una situación donde la segregación por nivel de estudios es superior a la segregación por nivel socioeconómico, en el caso de que el padre tenga estudios universitarios (que se corresponde al 12,07\% de estudiantes con mayor nivel socioeconómico).

El proceso de modelado multinivel para rendimiento en Matemática, como variable independiente, muestra resultados análogos a los encontrados anteriormente. 
Efectivamente, para todos los grupos minoritarios equivalentes la segregación escolar por nivel socioeconómico explica mayor cantidad de varianza del modelo que la segregación escolar por nivel de estudios (cuadro 5).

También es llamativo observar que la incidencia en el rendimiento en Matemática es mucho mayor para los grupos minoritarios de mayor nivel de estudios o mayor nivel socioeconómico. Siendo la menor incidencia (siempre controlado por nivel socioeconómico del estudiante) para la segregación con ambos padres sin estudios completos de primaria y con 10,6\% de estudiantes con familias de menos nivel socioeconómico.

Cuadro 4. Resumen de resultados de los procesos de Modelado multinivel sobre la incidencia de la segregación escolar por nivel educativos del padre y de la madre del estudiante y de la segregación por nivel socioeconómico en el rendimiento en Matemática

\begin{tabular}{lc|lc}
\hline \multicolumn{1}{c|}{ Segregación POR NIVEL DE ESTUdios } & $\begin{array}{c}\text { \% VAR. } \\
\text { EXPLICADA }\end{array}$ & $\begin{array}{c}\text { SEGREGACIÓN } \\
\text { SOCIOECONÓMICA }\end{array}$ & $\begin{array}{c}\text { \% VAR. } \\
\text { EXPLICADA }\end{array}$ \\
\hline Madre y padre sin estudios com. de Primaria & 1,05 & P10,64 & 2,75 \\
Padre sin estudios completos de Primaria & 1,64 & P16,35 & 3,32 \\
Madre sin estudios completos de Primaria & 1,26 & P20,28 & 3,76 \\
\hline Madre y padre hasta Primaria o menos & 3,53 & P25,19 & 4,38 \\
Padre con Primaria o menos & 2,11 & P32,10 & 5,48 \\
Madre hasta Primaria o menos & 1,63 & P37,18 & 6,30 \\
\hline Madre estudios universitarios acabados & 8,58 & P86,70 & 8,60 \\
Padre estudios universitarios acabados & 8,37 & P87,93 & 8,92 \\
Madre y padre con estudios universitarios ac. & 7,27 & P92,25 & 7,29 \\
\hline
\end{tabular}

Fuente: Elaboración propia a partir de datos de SABER 2016.

\section{Discusión y conclusiones}

Este estudio ha aportado sólidas y estables evidencias acerca de los dos objetivos planteados. Así, por una parte, ha encontrado que la segregación escolar por nivel socioeconómico es mayor que la segregación escolar por nivel de estudios del padre y la madre para todas las consideraciones de grupos minoritarios. De esta forma, parece ser que genera más segregación el nivel económico de las familias, su renta, que el nivel de estudios del padre o de la madre. Por otra parte, esta investigación ha hallado evidencias de que la segregación escolar por nivel socioeconómico incide más en el rendimiento académico de los estudiantes, tanto en Lengua como en Matemática, que la segregación por nivel de estudios de las familias.

Con ello se puede concluir que, efectivamente, es más adecuado el uso del nivel socioeconómico que el nivel de estudios de los padres para medir la segregación escolar: por ser este índice el que está generando mayor segregación escolar y por ser el que incide en mayor medida en el aprendizaje de los y las estudiantes.

De las pocas investigaciones que han usado el nivel de estudios de las familias, los resultados encontrados son coherentes. Por ejemplo, Mancebón-Torrubia y PérezXiménez (2010) informaron índices de segregación más bajos de segregación según el nivel de estudios de los progenitores que los obtenidos según el tipo de trabajo o el nivel socioeconómico y cultural de las familias. En cambio, Bartholo (2014) en Brasil o Böhlmark, Holmlund y Lindahl (2016) en Suecia, hallaron una mayor segregación por nivel de estudios de los padres. Estas diferencias, pueden ser explicadas por el trabajo de Yang Hansen y Gustafsson (2016b), que encuentran que la influencia de los estudios de 
los padres o del nivel socioeconómico en el rendimiento de los estudiantes es diferente en cada país, por lo que es normal que sea diferente la segregación escolar y su influencia en el aprendizaje estudiantil.

En todo caso, tal y como demuestra Lee, Zhang y Stankov (2019), una medida compuesta que incorpore variables de ocupación de los progenitores, ingresos familiares, nivel educativo del padre y de la madre e incluso algún tipo variable sobre salud familiar es capaz de explicar la varianza del aprendizaje de los estudiantes mucho mejor que estas variables tomadas individualmente. De esta forma, es posible concluir que la mejor variable criterio para estimar la segregación escolar sería un índice compuesto que recogiera, al menos, información del nivel socioeconómico y del nivel cultural de las familias. La buena noticia es que esa es la opción más habitual en las investigaciones sobre segregación, al menos en las que usan datos de evaluaciones internacionales y muchas de las nacionales.

Pero la utilidad de estos resultados va más allá de una cuestión puramente metodológica. Esta investigación, al encontrar que el nivel socioeconómico de las familias, pero también su nivel cultural, generan segregación escolar y tienen una clara incidencia sobre el rendimiento de los estudiantes, apunta a la necesidad de que las medidas para luchar contra la segregación tengan en cuenta no solo los ingresos familiares, también el nivel de estudios de los padres.

El presenta trabajo tiene varias fortalezas de carácter metodológico. Destaca el hecho de usar datos censales, lo que evita cualquier sesgo por muestreo. Contar con datos de 646.413 estudiantes es claramente un punto fuerte de este trabajo. Además, los datos son internamente coherentes al usar dos índices para medir la segregación y dos materias para analizar su influencia. Contar con datos de un país, como Colombia, con una alta segregación puede haber favorecido una mayor visibilidad en los contrastes, pero no hay razones para pensar que hubieran sido muy diferentes con otro contexto. En todo caso, habría que confirmar estos resultados para otras realidades.

Este trabajo ha intentado contribuir a la necesaria reflexión metodológica y técnica para estimar la segregación escolar. En todo caso, nos queda mucho por saber. Sería importante, por ejemplo, trabajar para conseguir un consenso entre los equipos de investigación para asumir un concepto de segregación y su medida y profundizar en él. Solo en la medida que desde la investigación podamos denunciar la situación de inequidad de nuestros sistemas educativos, aportando datos claros sobre la situación, sus consecuencias y causas, estaremos aportando ideas para que cambie esa realidad.

Decía Desmond Tutu, premio nobel de la paz, "Si eres neutral en situaciones de injusticia, has elegido el lado del opresor". Esta frase contundente se aplica a la perfección a la investigación educativa. Si nos limitamos a describir una realidad de forma neutral, sin contribuir a su transformación, estamos colaborando en su legitimación, estamos ayudando a los poderosos que quieren que nada cambie porque la situación les favorece. La investigación social tiene como primera misión denunciar situaciones de injusticia, y, como segunda, aportar datos que ayuden a comprenderlas y a tomar las adecuadas decisiones para transformarlas. La segregación escolar es un indicador de un sistema educativo injusto. En la medida que la investigación puede aportar datos útiles para acabar con ella, estará contribuyendo a una sociedad más justa y equitativa. 


\section{Referencias}

Bartholo, T. L. (2013). Measuring between-school segregation in an open enrollment system: the case of Rio de Janeiro. Journal of School Choice, 7(3), 353-371. https://doi.org/10.1080/15582159.2013.808937

Bartholo, T. L. (2014). Segregação escolar na cidade do Rio de Janeiro: Análise da movimentação de estudantes. Estudos em Avaliação Educacional, 25(58), 242-271. https://doi.org/10.18222/eae255820142927

Benito, R. y González-Baetbó, I. (2007). Processos de segregació escolar a Catalunya. Mediterrània.

Bodovski, K. (2010). Parental practices and educational achievement: Social class, race, and habitus. British Journal of Sociology of Education, 31(2), 139-156. https://doi.org/10.1080/01425690903539024

Böhlmark, A., Holmlund, H. y Lindahl, M. (2016). Parental choice, neighbourhood segregation or cream skimming? An analysis of school segregation after a generalized choice reform. Journal of Population Economics, 29(4), 1155-1190. https://doi.org/10.1007/s00148-016-0595-y

Bonal, X., Zancajo, A. y Scandurra, R. (2019). Residential segregation and school segregation of foreign students in Barcelona. Urban Studies, 56(15), 3251-3273. https://doi.org/10.1177/0042098019863662

Bourdieu, P. (1973). Cultural reproduction and social reproduction. En R. Brown (Ed.), Knowledge, education, and cultural change: Papers in the sociology of education (pp. 71-84). Tavistock.

Brese, F. y Mirazchiyski, P. (2013). Measuring students' family background in largescale international education studies. IEA-ETS Research Institute.

Coleman, J. S. (1975). Trends in school segregation, 1968-73. Urban Institute.

Croxford, L. y Raffe, D. (2013). Differentiation and social segregation of UK higher education, 1996-2010. Oxford Review of Education, 39(2), 172-192. https://doi.org/10.1080/03054985.2013.784193

Davies, S. y Rizk, J. (2018). The three generations of cultural capital research: A narrative review. Review of Educational Research, 88(3), 331-365. https://doi.org/10.3102/0034654317748423

Davis-Kean, P. E. (2005). The influence of parent education and family income on child achievement: The indirect role of parental expectations and the home environment. Journal of Family Psychology, 19(2), 294-304. https://doi.org/10.1037/0893-3200.19.2.294

Desforges, C. y Abouchaar, A. (2003). The impact of parental involvement, parental support and family education on pupil achievement and adjustment. DfES.

Erola, J., Jalonen, S. y Lehti, H. (2016). Parental education, class and income over early life course and children's achievement. Research in Social Stratification and Mobility, 44, 33-43. https://doi.org/10.1016/j.rssm.2016.01.003

Fajardo Bullón, F., Maestre Campos, M., Felipe Castaño, E., León del Barco, B. y Polo del Río, M. I. (2017). Análisis del rendimiento académico de los alumnos de educación secundaria obligatoria según las variables familiares. Educación XXI, 2O(1), 209-232.

https://doi.org/ 10.5944/educxx 1.17509

Fiel, J. E. y Zhang, Y. (2018). Three dimensions of change in school segregation: A grade-periodcohort analysis. Demography, 55(1), 33-58. https://doi.org/10.1007/s13524-017-0632-9 
Fuller, B., Kim, Y., Galindo, C., Bathia, S., Bridges, M., Duncan, G. J. y García Valdivia, I. (2019). Worsening school segregation for Latino children? Educational Researcher, 48(7), 407-420. https://doi.org/10.3102/0013189X19860814

Gorard, S. y See, B. (2013). Overcoming disadvantage in education. Routledge. https://doi.org/10.4324/9780203741665

Gorard, S. y Siddiqui, N. (2018). Grammar schools in England: A new analysis of social segregation and academic outcomes. British Journal of Sociology of Education, 39(7), 909-924. https://doi.org/10.1080/01425692.2018.1443432

Gorard, S. y Taylor, C. (2002). What is segregation? A comparison of measures in terms of 'strong'and 'weak'compositional invariance. Sociology, 36(4), 875-895. https://doi.org/10.1177/003803850203600405

Gramberg, P. (1998). School segregation: The case of Amsterdam. Urban Studies, 35(3), 547-564. https://doi.org/10.1080/0042098984907

Gustafsson, J. E., Yang Hansen, K. y Rosén, M. (2013). Effects of home background on student achievement in reading, mathematics, and science at the fourth grade. En M. O. Martin y I. V. S. Mullis (Eds.), TIMSS and PIRLS 2011: Relationships among reading, mathematics, and science achievement at the fourth grade. Implications for early learning (pp. 183-289). TIMSS \& PIRLS International Study Center, Boston College.

Hutchens, R. (2001). Numerical measures of segregation: Desirable properties and their implications. Mathematical Social Sciences, 42(1), 13-29. https://doi.org/10.1016/SO165-4896(00)00070-6

Hutchens, R. (2004). One measure of segregation. International Economic Review, 45(2), 555-578. https://doi.org/10.1111/j.1468-2354.2004.00136.x

Ivaniushina, V., Makles, A. M., Schneider, K. y Alexandrov, D. (2019). School segregation in St. Petersburg. The role of socioeconomic status. Education Economics, 27(2), 166-185. https://doi.org/10.1080/09645292.2018.1538408

Janmaat, J. G. (2020). School social segregation and social inequalities in political engagement among 16 to 20 year olds in fourteen countries. Research Papers in Education. https://doi.org/10.1080/02671522.2020.1789716

Karsten, S. (2010). School segregation. En OECD, Equal opportunities? The labour market integration on the children of immigrants (pp. 193-209). OECD Publishing. https://doi.org/10.1787/9789264086395-en

Kornhall, P. y Bender, G. (2019). School segregation in Sweden: Evidence from the local level. NESET.

Krüger, N. (2019). La segregación por nivel socioeconómico como dimensión de la exclusión educativa. 15 años de evolución en América Latina. Education Policy Analysis Archives, 27(8). https://doi.org/10.14507/epaa.27.3577

Lee, J., Zhang, Y. y Stankov, L. (2019) Predictive validity of SES measures for student achievement. Educational Assessment, 24(4), 305-326. https://doi.org/10.1080/10627197.2019.1645590

Maloutas, T., Hadjiyanni, A., Kapella, A., Spyrellis, S. N. y Valassi, D. (2013, agosto). Education and social reproduction: The impact of social origin, school segregation and residential segregation on educational performance in Athens. Comunicación presentada en RC21 (ISA) Conference on 'Resourceful Cities' Resourceful Cities, Berlin.

Mancebón-Torrubia, M. J. y Pérez-Ximénez, D. (2010). Una valoración del grado de segregación socioeconómica existente en el sistema educativo español. Un análisis por comunidades autónomas a partir de PISA 2006. Regional and Sectoral Economic Studies, 10(3), 129-148. 
Massey, D. S. y Denton, N. A. (1988). Hypersegregation in U.S. metropolitan areas: Black and Hispanic segregation along five dimensions. Demography, 26, 373- 391.

https://doi. org/10.2307/2061599

Murillo, F. J. (2016). Midiendo la segregación escolar en América Latina. Un análisis metodológico utilizando el TERCE. REICE. Revista Iberoamericana sobre Calidad, Eficacia y Cambio en Educación, 14(4), 33-60. https://doi.org/10.15366/reice2016.14.4.002

Murillo, F. J. y Martínez-Garrido, C. (2018). Magnitud de la segregación escolar por nivel socioeconómico en España y sus Comunidades Autónomas y comparación con los países de la Unión Europea. RASE. Revista de Sociología de la Educación, 11(1), 37-58. https://doi.org/10.7203/RASE.11.1.10129

Murillo, F. J. y Martínez-Garrido, C. (2019). Perfiles de segregación escolar por nivel socioeconómico en España y sus Comunidades Autónomas. RELIEVE, 25(1), art. 1. https://doi.org/10.7203/relieve.25.1.12917

Murillo, F. J. y Román, M. (2011). ¿La escuela o la cuna? Evidencias sobre su aportación al rendimiento de los estudiantes de América Latina. Estudio multinivel sobre la estimación de los efectos escolares. Profesorado. Revista de Curriculum y Formación de Profesorado, 15(3), 27-53.

Murillo, F. J., Duk, C. y Martínez-Garrido, C. (2018). Evolución de la segregación socioeconómica de las escuelas de América Latina. Estudios Pedagógicos, 44(1), 157-179.

https://doi.org/10.4067/SO718-07052018000100157

Murillo, F. J., Martínez-Garrido, C. y Belavi, G. (2017). Segregación escolar por origen nacional en España. OBETS. Revista de Ciencias Sociales, 12(2), 395-432. https://doi.org/10.14198/OBETS2017.12.2.04

Nordin, M. (2013). Immigrant school segregation in Sweden. Population Research and Policy Review, 32(3), 415-435. https://doi.org/10.1007/s $11113-013-9271-\mathrm{Z}$

OECD. (2010). PISA 2009 results: Overcoming social background. Equity in learning opportunities and outcomes. OECD Publishing. https://doi.org/10.1787/9789264091504-en

OECD. (2019). PISA 2018 results: Where all students can succeed. OECD Publishing. https://doi.org/10.1787/b5fd1b8f-en

Ortiz, E. A. y Dehon, C. (2008). What are the factors of success at university? A case study in Belgium. Economic Studies, 54(2), 121-148. https://doi.org/10.1093/cesifo/ifno12

Santiago, M. (2019). A framework for an interdisciplinary understanding of Mexican American school segregation. Multicultural Education Review, 11(2), 69-78.

https://doi.org/10.1080/2005615X.2019.1615246

Siddiqui, N. (2017). Parental education as a determinant of school choice: A comparative study of school types in Pakistan. Research in Education, 99(1), 3-18.

https://doi.org/10.1177/0034523717725862

Silles, M. A. (2011). The intergenerational effects of parental schooling on the cognitive and noncognitive development of children. Economics of Education Review, 30(2), 258-268. https://doi.org/10.1016/j.econedurev.2010.09.002

Sirin, S. R. (2005). Socioeconomic status and academic achievement: A meta-analytic review of research. Review of Educational Research, 75(3), 417-453. https://doi.org/10.3102/00346543075003417

White, K. R. (1982). The relation between socioeconomic status and academic achievement. Psychological Bulletin, 91,461-481. https://doi.org/10.1037/0033-2909.91.3.461 
Wilson, W. J. (1987). The truly disadvantaged: The inner city, the underclass, and public policy. University of Chicago Press.

Yang Hansen, K. y Gustafsson, J. E. (2016). Causes of educational segregation in Sweden: School choice or residential segregation. Educational Research and Evaluation, 22(1-2), 23-44. https://doi.org/10.1080/13803611.2016.1178589

Yang Hansen, K. y Gustafsson, J. E. (2016b). Determinants of country differences in effects of parental education on children's academic achievement. Large-scale Assessments in Education, 4(11). https://doi.org/10.1186/s40536-016-0027-1

Zhou, Y., Cai, T. y Wang, D. (2016). Social segregation in Hong Kong's schools: 2000-2012. Chinese Sociological Review, 48(3), 248-270.

https://doi.org/10.1080/21620555.2016.1166340 


\section{Anexo}

Cuadro A1. Resultados del proceso de Modelado Multinivel sobre la incidencia de la segregación escolar por nivel educativos del padre y de la madre del estudiante en el rendimiento en Lengua

\begin{tabular}{|c|c|c|c|c|c|c|c|c|c|c|}
\hline & MODELO 1 & MODELO 2A & MODELO 2B & MODELO 2C & MODELO 2D & MODELO 2E & & & & \\
\hline & B (EE) & B (EE) & B (EE) & B (EE) & B (EE) & B (EE) & & & & \\
\hline \multicolumn{11}{|l|}{ Parte fija } \\
\hline Intercepto & $435,26(1,02)$ & $439,29(1,05)$ & $440,07(1,05)$ & $439,58(1,05)$ & $439,83(1,06)$ & $44 \cdot 1,12(1,05)$ & $440,66(1,00)$ & $434,60(1,00)$ & $343,66(1,00)$ & $434,34(1,00)$ \\
\hline SES & $1,56(0,02)$ & $1,53(0,02)$ & $1,52(0,02)$ & $1,52(0,02)$ & $1,52(0,02)$ & $1,51(0,02)$ & $1,51(0,02)$ & $1,49(0,02)$ & $1,49(0,02)$ & $1,50(0,02)$ \\
\hline Seg e1 & & $\begin{array}{c}-154268,72 \\
(9693,39)\end{array}$ & & & & & & & & \\
\hline Seg. e2 & & & $\begin{array}{r}-217407,45 \\
(11329,90)\end{array}$ & & & & & & & \\
\hline Seg e3 & & & & $\begin{array}{r}-208154,27 \\
(11771,29)\end{array}$ & & & & & & \\
\hline Seg e4 & & & & & $\begin{array}{c}-185640,66 \\
(10804,01)\end{array}$ & & & & & \\
\hline Seg e 5 & & & & & & $\begin{array}{r}-259786,34 \\
(11666,18)\end{array}$ & & & & \\
\hline Seg e6 & & & & & & & $\begin{array}{r}-247334,09 \\
(11843,37)\end{array}$ & & & \\
\hline Seg e7 & & & & & & & & $\begin{array}{c}175397,31 \\
(4621,56)\end{array}$ & & \\
\hline Seg e8 & & & & & & & & & $\begin{array}{c}189894,31 \\
(4878,72)\end{array}$ & \\
\hline Seg e9 & & & & & & & & & & $\begin{array}{c}118015,58 \\
(344,11) \\
\end{array}$ \\
\hline \multicolumn{11}{|l|}{ Parte aleatoria } \\
\hline Entre escuelas & 3508,64 & 3443,19 & $34 \cdot 10,97$ & 3426,32 & 3434,09 & 3375,11 & 3394,61 & 3082,78 & 3064,32 & 3151,91 \\
\hline Entre alumnos & 6438,49 & 6437,44 & 6437,40 & 6438,26 & 6437,28 & 6437,04 & 6437,25 & 6440.31 & 6440.35 & 6440,01 \\
\hline $\begin{array}{l}\text { \% var explicada de } \\
\text { escuela }\end{array}$ & 0,00 & 1,87 & 2,78 & 2,35 & 2,12 & 3,81 & 3,25 & 12,14 & 12,66 & 10,17 \\
\hline
\end{tabular}

Fuente: Elaboración propia a partir de datos de SABER 2016. 


\section{F. J. Murillo y R. Graña}

Cuadro A2. Resultados del proceso de Modelado Multinivel sobre la incidencia de la segregación escolar por nivel socioeconómico en el rendimiento en Lengua

\begin{tabular}{|c|c|c|c|c|c|c|c|c|c|c|}
\hline & MODELO 1 & MODELO 2A & MODELO 2B & MODELO 2C & MODELO 2D & MODELO 2E & & & & \\
\hline & B (EE) & B (EE) & B (EE) & B (EE) & B (EE) & B (EE) & & & & \\
\hline \multicolumn{11}{|l|}{ Partefija } \\
\hline Intercepto & $435,26(1,02)$ & $440,46(1,05)$ & $441,46(1,05)$ & $442,15(1,05)$ & $442,86(1,06)$ & $444,15(1,06)$ & $444,97(1,06)$ & $434,08(1,00)$ & $494,11(1,00)$ & $434,28(1,00)$ \\
\hline SES & $1,56(0,02)$ & $1,51(0,02)$ & $1,50(0,02)$ & $1,50(0,02)$ & $1,49(0,02)$ & $1,48(0,02)$ & $1,48(0,02)$ & $1,48(0,02)$ & $1,49(0,02)$ & $1,50(0,02)$ \\
\hline Seg c1 & & $\begin{array}{c}-118161,05 \\
(5431,20)\end{array}$ & & & & & & & & \\
\hline Seg. c2 & & & $\begin{array}{c}-155596,95 \\
(6453,03)\end{array}$ & & & & & & & \\
\hline Sep c3 & & & & $\begin{array}{c}-180247,58 \\
(6980,33)\end{array}$ & & & & & & \\
\hline Seg c 4 & & & & & $\begin{array}{c}-208966,38 \\
(7477,70)\end{array}$ & & & & & \\
\hline Seg c 5 & & & & & & $\begin{array}{c}-246132,91 \\
(7909,28)\end{array}$ & & & & \\
\hline Seg c6 & & & & & & & $\begin{array}{c}-268774,91 \\
(8051,19)\end{array}$ & & & \\
\hline Seg c7 & & & & & & & & $\begin{array}{l}154155,72 \\
/ 3932,54)\end{array}$ & & \\
\hline Seg c8 & & & & & & & & & $\begin{array}{l}144136,42 \\
(3744,06)\end{array}$ & \\
\hline Seg c9 & & & & & & & & & & $\begin{array}{l}104158,73 \\
(2978,81) \\
\end{array}$ \\
\hline \multicolumn{11}{|l|}{ Parte aleatoria } \\
\hline Entre escuelas & 3508,64 & 3390,78 & 3360,60 & 3338,05 & 3307,84 & 3257,07 & 3217,82 & 3061,65 & 3075,94 & 3145,39 \\
\hline Entre alumnos & 6438,49 & 6437,49 & 6438,20 & 6437,44 & 6437,40 & 6437,47 & 6438,35 & 6440,86 & 6440,77 & 6440,35 \\
\hline $\begin{array}{l}\text { \% var explicada de } \\
\text { escuela }\end{array}$ & 0,00 & 3,36 & 4,22 & 4,86 & 5,72 & 7,17 & 8,29 & 12,74 & 12,33 & 10,35 \\
\hline
\end{tabular}

Fuente: Elaboración propia a partir de datos de SABER 2016. 
Cuadro A3. Resultados del proceso de Modelado Multinivel sobre la incidencia de la segregación escolar por nivel de estudios de los padres en el rendimiento en Matemática

\begin{tabular}{|c|c|c|c|c|c|c|c|c|c|c|}
\hline & MODELO 1 & MODELO 2A & MODELO 2B & MODELO 2C & MODELO 2D & MODELO 2E & & & & \\
\hline & B (EE) & B (EE) & B (EE) & B (EE) & B (EE) & B (EE) & & & & \\
\hline \multicolumn{11}{|l|}{ Partefija } \\
\hline Intercepto & $\begin{array}{c}433,55 \\
(1,03)\end{array}$ & $436,65(1,06)$ & $437,33(1,06)$ & $436,80(1,06)$ & $436,77(1,07)$ & $437,99(1,07)$ & $437,50(1,07)$ & $432,90(1,01)$ & $432,94(1,01)$ & $432,68(1,02)$ \\
\hline SES & $1,64(0,02)$ & $1,62(0,02)$ & $1,61(0,02)$ & $1,61(0,02)$ & $1,61(0,02)$ & $1,60(0,02$ & $1,61(0,02)$ & $1,58(0,02)$ & $1,58(0,02)$ & $1,59(0,02)$ \\
\hline Seg e1 & & $\begin{array}{r}-124525,45 \\
(10305,05)\end{array}$ & & & & & & & & \\
\hline Seg. e2 & & & $\begin{array}{r}-178786,89 \\
(12042,24)\end{array}$ & & & & & & & \\
\hline Seg e3 & & & & $\begin{array}{r}-164713,83 \\
(12526,02)\end{array}$ & & & & & & \\
\hline Seg e4 & & & & & $\begin{array}{r}-137715,00 \\
(11484,56)\end{array}$ & & & & & \\
\hline Seg e 5 & & & & & & $\begin{array}{r}-205827,45 \\
(12435,48)\end{array}$ & & & & \\
\hline Seg e6 & & & & & & & $\begin{array}{r}-189517,52 \\
(12617,61)\end{array}$ & & & \\
\hline Seg e7 & & & & & & & & $\begin{array}{c}156491,56 \\
(4976,49)\end{array}$ & & \\
\hline Seg e8 & & & & & & & & & $\begin{array}{c}168939 \\
(5258,76)\end{array}$ & \\
\hline Seg e9 & & & & & & & & & & $\begin{array}{l}105856,73 \\
(3690,88) \\
\end{array}$ \\
\hline \multicolumn{11}{|l|}{ Parte aleatoria } \\
\hline Entre escuelas & 3950,79 & 3909,44 & 3886,01 & 3901,03 & 3811,52 & 3867,47 & 3886,21 & 3611,17 & 3598,22 & 3662,63 \\
\hline Entre alumnos & 6274,39 & 6273,80 & 6273,78 & 6273,79 & 6273,74 & 6273,85 & 6273,69 & 6275,98 & 6276,02 & 6275,78 \\
\hline $\begin{array}{l}\% \text { var explicada de } \\
\text { escuela }\end{array}$ & 0,00 & 1,05 & 1,64 & 1,26 & 3,53 & 2,11 & 1,63 & 8,60 & 8,92 & 7,29 \\
\hline
\end{tabular}

Fuente: Elaboración propia a partir de datos de SABER 2016. 


\section{F. J. Murillo y R. Graña}

Cuadro A4. Resultados del proceso de Modelado Multinivel sobre la incidencia de la segregación escolar por nivel socioeconómico en el rendimiento en

Matemática

\begin{tabular}{|c|c|c|c|c|c|c|c|c|c|c|}
\hline & MODELO 1 & MODELO 2A & MODELO 2B & MODELO 2C & MODELO 2D & MODELO 2E & & & & \\
\hline & B (EE) & B (EE) & B (EE) & B (EE) & B (EE) & B (EE) & & & & \\
\hline \multicolumn{11}{|l|}{ Parte fija } \\
\hline Intercepto & $433,55(1,03)$ & $438,21(1,06)$ & $439,08(1,06)$ & $439,66(1,06)$ & $440,36(1,07)$ & $441,44 \cdot(1,07)$ & $442,17(1,07)$ & $432,49(1,01)$ & $432,52(1,01)$ & $432,66(1,02)$ \\
\hline SES & $1,64 \cdot(0,02)$ & $1,60(0,02)$ & $1,59(0,02)$ & $1,59(0,02)$ & $1,58(0,02)$ & $1,58(0,02)$ & $1,57(0,02)$ & $1,58(0,02)$ & $1,58(0,02)$ & $1,59(0,02)$ \\
\hline Seg c1 & & $\begin{array}{c}-110317,87 \\
(5773,19)\end{array}$ & & & & & & & & \\
\hline Seg. c2 & & & $\begin{array}{c}-144614,55 \\
(6861,52)\end{array}$ & & & & & & & \\
\hline Sep c3 & & & & $\begin{array}{c}-166393,27 \\
(7426,89)\end{array}$ & & & & & & \\
\hline Seg c4 & & & & & $\begin{array}{c}-192184,28 \\
(7962,45)\end{array}$ & & & & & \\
\hline Seg c5 & & & & & & $\begin{array}{c}-226805,59 \\
(8434,22)\end{array}$ & & & & \\
\hline Seg c6 & & & & & & & $\begin{array}{c}-247477,19 \\
(8598,00)\end{array}$ & & & \\
\hline Seg c7 & & & & & & & & $\begin{array}{c}139964,19 \\
(4248,27)\end{array}$ & & \\
\hline Seg c8 & & & & & & & & & $\begin{array}{l}125675,68 \\
(4040,56)\end{array}$ & \\
\hline Seg c9 & & & & & & & & & & $\begin{array}{l}92258,68 \\
(3199,87) \\
\end{array}$ \\
\hline \multicolumn{11}{|l|}{ Parte aleatoria } \\
\hline Entre escuelas & 3950,79 & 3842,18 & 3819,53 & 3802,13 & 3777,94 & 3734,45 & 3702,01 & 3611,64 & 3620,13 & 3663,56 \\
\hline Entre alumnos & 6274,39 & 6273,99 & 6273,89 & 6273,87 & 6273,84 & 6273,90 & 6273,96 & 6276,22 & 6276,17 & 6275,93 \\
\hline $\begin{array}{l}\text { \% var explicada de } \\
\text { escuela }\end{array}$ & 0,00 & 2,75 & 3,32 & 3,76 & 4,38 & 5,48 & 6,30 & 8,58 & 8,37 & 7,27 \\
\hline
\end{tabular}

Fuente: Elaboración propia a partir de datos de SABER 2016. 


\section{Breve CV de los autores}

\section{F. Javier Murillo}

Director de la Cátedra UNESCO en Educación para la Justicia Social de la Universidad Autónoma de Madrid. Profesor titular del Área de Métodos de Investigación y Diagnóstico en Educación de la Universidad Autónoma de Madrid. Coordinador del grupo de Investigación Cambio Educativo para la Justicia Social (GICE). Secretario académico del Instituto de Derechos Humanos, Democracia y Cultura de Paz y No Violencia (DEMOSPAZ), de la UAM. Fue Coordinador General del Laboratorio Latinoamericano de Evaluación de la Calidad de la Educación (LLECE), de la UNESCO, y Director de Estudios del Centro de Investigación y Documentación Educativa (CIDE) del Ministerio de Educación de España. Es Coordinador de la Red Iberoamericana de Investigación sobre Cambio y Eficacia Escolar (RINACE), Director de REICE. Revista Iberoamericana sobre Calidad, Eficacia y Cambio en Educación, de la Revista Iberoamericana de Evaluación Educativa y de la Revista Internacional de Educación para la Justicia Social. Página personal: https://www.fjaviermurillo.es. ORCID ID: https://orcid.org/0000-0002-8003-4133. E-mail: javier.murillo@uam.es

\section{Raquel Graña Oliver}

Personal Técnico en Investigación de la Cátedra UNESCO en Educación para la Justicia Social de la Universidad Autónoma de Madrid. Máster en Calidad y Mejora de la Educación especializada en gestión y liderazgo escolar por la Universidad Autónoma de Madrid (España), Grado em Educación Infantil por la Universidad de Valladolid. Miembro del Grupo de Investigación Cambio Educativo para la Justicia Social, Universidad Autónoma de Madrid. En la actualidad está desarrollando su tesis doctoral sobre el tema de segregación escolar. ORCID ID: http://orcid.org/0000-0002-42238354.E-mail: raquel.granna@uam.es 